\section{Dark-field Illumination for Photographing Precipitin Bands in Gel}

\section{J. M. RUDGE}

From the Department of Bacteriology, University of Edinburgh

(RECEIVED FOR PUBLICATION AUGUST 3, 1960)

A variety of techniques for photographing precipitin bands in gel have been published, some using forms of dark-field illumination (Hunter, 1959), others using bright-field illumination (Dike, 1960). Dark-field lighting gives better visual contrast with faint unstained bands and has also been favoured for photographic work. Jackson (1959) compared various lighting

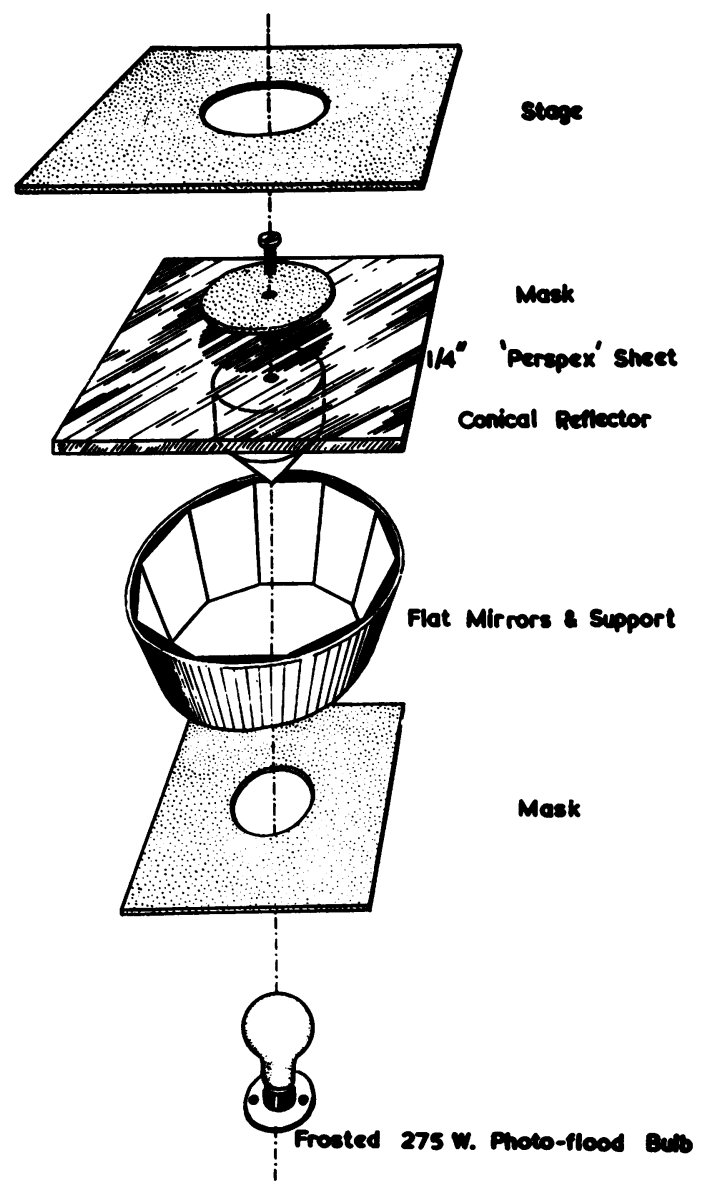

FIG. 1.- "Exploded" perspective drawing of the main components. systems and photographic techniques and found that, with unstained preparations, a dark-field method gave better results. With stained bands, the relative efficiency of the lighting methods was influenced by the colour of the stain employed; with an orange stain, dark-field lighting gave better results, with a blue stain good results were obtained by either method.

The influence of the angle of illumination on photographic contrast has been investigated by Hunter. He adopted a bilaterally symmetrical darkfield lighting system in which the Petri dish was illuminated from below by two $500 \mathrm{w}$. spotlights with their beams converging in a vertical plane and passing through the agar at $21^{\circ}$ to the normal. With complex serological systems, Hunter found that greatest separation of bands was obtained when they lay parallel to the light plane. It follows that uniform separation of bands in asymmetrical patterns cannot be achieved by this method. In comparative studies, where it is important to ensure uniform separation of bands and to record faithfully their relative density, radially symmetrical lighting would appear preferable.
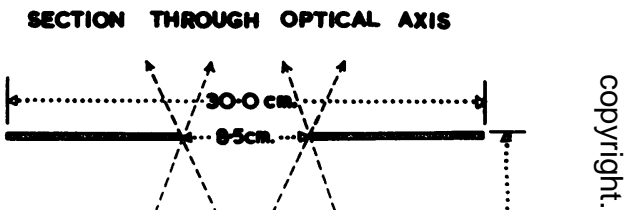
Ouchterlony (1958), Schutz (1958), and Reed (1960) have described radially symmetrical dark-field systems in which a ring-shaped lamp, or a masked $x$-ray viewing box, was used as a light source. An alternative method, which is both versatile and inexpensive, has been used routinely in this department for the past year. The equipment is designed to give uniform dark-field illumination at $21^{\circ}$ to the normal for Petri dishes up to $9 \mathrm{~cm}$. in diameter. Only one light source is required. Electronic flash or a variety of tungsten lamps can be interchanged readily where use of a variety of photographic emulsions makes this desirable.

The main components of the apparatus are illustrated in Fig. 1, and the light path and dimensions are shown in Fig. 2.

The conical reflector was turned from 3 in. brass rod, the base drilled and tapped, and the reflecting surface carefully polished to remove all trace of tool marks. The 10 flat reflectors were cut from $3 \frac{1}{4}$ by $3 \frac{1}{4}$ in. lantern slide cover glasses. The conical and flat reflecting surfaces were then coated with aluminium in a high vacuum evaporation unit of the kind used in conjunction with an electron microscope. (Where this equipment is not available, several alternatives can be adopted. The conical reflector can be chromiumplated or turned from any colourless metal which will take a high polish and, where necessary, the surface protected with a thin coat of colourless varnish. The flat reflectors can be silvered or cut from thin glass mirror.)

The supporting collar for the flat reflectors was cut from 18 gauge sheet metal, and the ends were drilled and bent upwards at right angles. The arc was then bent to form a truncated cone, and the ends bolted together.

The flat reflectors were mounted by placing them face downwards in a flat arc with their adjacent edges parallel and slightly apart. These edges were bound together by strips of adhesive tape, and then the two ends of the arc were brought together and joined in the same fashion.

The stage and masks were painted matt black on both sides, and the various components were mounted coaxially in a suitable framework. A wall-mounted enlarger fitted with a copying head and a $13.5 \mathrm{~cm}$. lens was used as the camera; it was therefore convenient to arrange the stage at bench level. With a 275 w. photoflood bulb mounted at floor level, uniform exposures of $10 \mathrm{sec}$. at $f / 8$ were required for Ilford G.50 ortho half-tone backed plates with normal development in ID 13 developer.

Before photographing precipitin bands the agar was carefully washed and covered with distilled water to prevent undesirable reflections from the sides of the

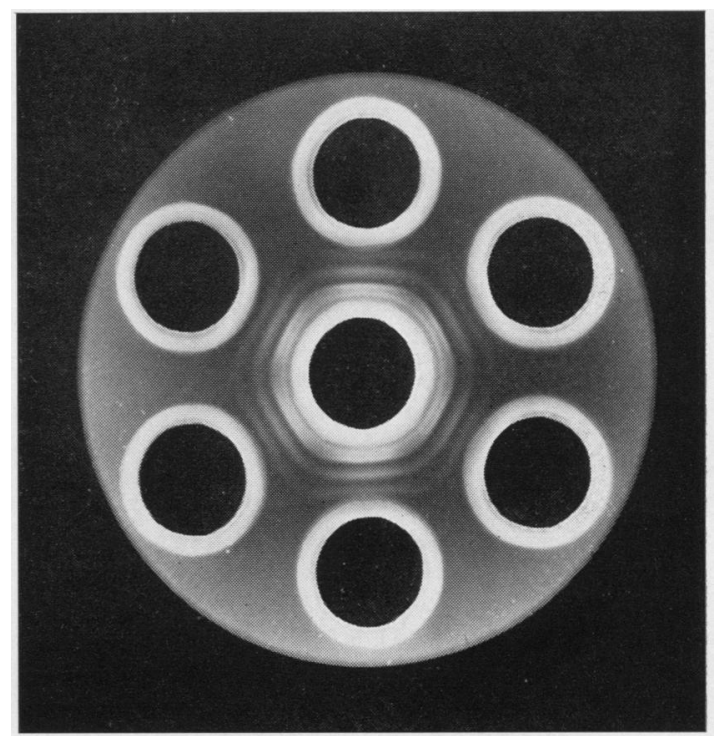

Fig. 3.-Photograph of a radially symmetrical serological system showing uniform lighting of precipitin bands. The six peripheral wells were filled with sonic-disintegrated Leptospira icterohaemorrhagiae; the central well contained the homologous antiserum.

wells. Scratches on the underside of the Petri dish were hidden by mounting the dish in a small pool of glycerol on a sheet of optically flat glass.

Where photographs of complex serological systems containing faint but adequately resolved bands are intended for publication it is suggested that the print should be prepared from a reversed negative. With such a photograph-showing dark precipitin bands against a pale background-there is less chance of delicate bands being obscured by ink spreading in the final illustration. Cross-printing of negatives also offers further opportunity for controlling contrast, although negatives prepared with the apparatus described here printed satisfactorily on normal grades of photographic paper.

The equipment was constructed in the course of studies financed in part by a New Zealand national research fellowship. I am indebted to Mr. G. A. Wilson for technical assistance.

\section{REFERENCES}

Dike, G. W. R. (1960). J. clin. Path., 13, 87.

Hunter, J. R. (1959). Nature (Lond.), 183, 1283. Jackson, R. (1959). J. biol. photogr. Ass., 27, 133. Ouchterlony, Ö. (1958). Progr. Allergy, 5, 1.

Reed, F. C. (1960). Amer. J. clin. Path., 33, 364 Schutz, J. N. (1958). J. biol. photogr. Ass., 26, 159. 